\title{
Correction to: The postoperative shorter meniscal width was the risk factor of lateral meniscal extrusion in the middle portion for juvenile and adolescent knees with discoid lateral meniscus
}

\author{
Tomoharu Mochizuki $^{1}$ (1) $\cdot$ Osamu Tanifuji ${ }^{1} \cdot$ Satoshi Watanabe ${ }^{2} \cdot$ Takashi Sato $^{2} \cdot$ Naoto Endo $^{1}$
}

Published online: 20 August 2020

(c) European Society of Sports Traumatology, Knee Surgery, Arthroscopy (ESSKA) 2020

\section{Correction to: \\ Knee Surgery, Sports Traumatology, Arthroscopy \\ https://doi.org/10.1007/s00167-020-06188-x}

Authors would like to correct the errors in figure legend and figures of Figs. 1 and 2. Correct version of Figs. 1 and 2 updated here.

The original article can be found online at https://doi.org/10.1007/ s00167-020-06188-x.

Tomoharu Mochizuki

tommochi121710@gmail.com

Osamu Tanifuji

tanifuji06242000@yahoo.co.jp

Satoshi Watanabe

wwsatoshiww@gmail.com

Takashi Sato

takuukat2032@gmail.com

Naoto Endo

endless@med.niigata-u.ac.jp

1 Division of Orthopedic Surgery, Department of Regenerative and Transplant Medicine, Niigata University Graduate School of Medical and Dental Science, 1-757 Asahimachi-dori Chuo-ku, Niigata, Niigata 951-8510, Japan

2 Department of Orthopaedic Surgery, Niigata Medical Center, 3-27-11, Kobari, Nishi-ku, Niigata 950-2022, Japan
Publisher's Note Springer Nature remains neutral with regard to jurisdictional claims in published maps and institutional affiliations.

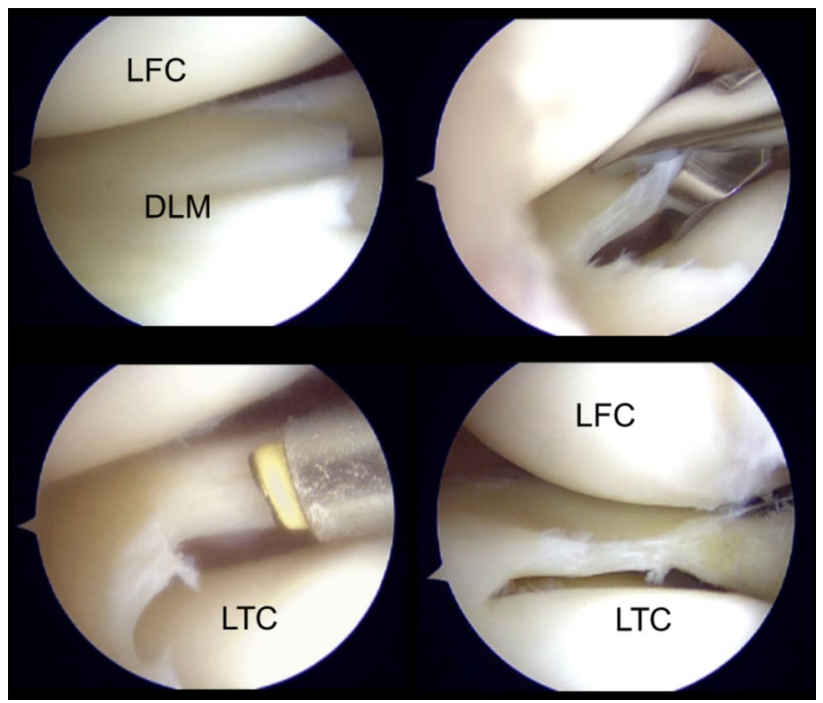

Fig. 1 Arthroscopic saucerization alone. After partial meniscectomy, the central margin was treated by a radio frequency to prevent regeneration of meniscus. $L F C$ lateral femoral condyle, $D L M$ discoid lateral meniscus, $L T C$ lateral tibial condyle 


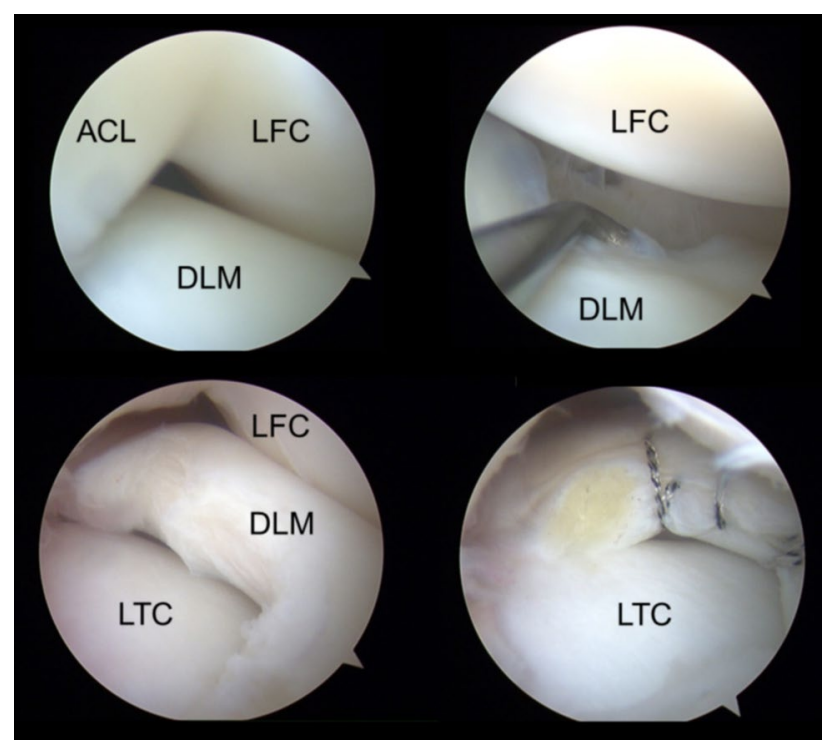

Fig. 2 Arthroscopic saucerization with meniscal repair. In this case, discoid lateral meniscus (DLM) showed the tear of the posterior meniscocapsular junction (anterocentral type) in the upper right figure. Following minimum partial meniscectomy in the lower left figure, the meniscus was repaired by various suture techniques. The remained meniscus was translated peripherally by suturing like the lower right figure because of the discontinuity and inhomogeneity of the collagen. $L F C$ lateral femoral condyle, $D L M$ discoid lateral meniscus, $A C L$ anterior cruciate ligament, $L T C$ lateral tibial condyle 